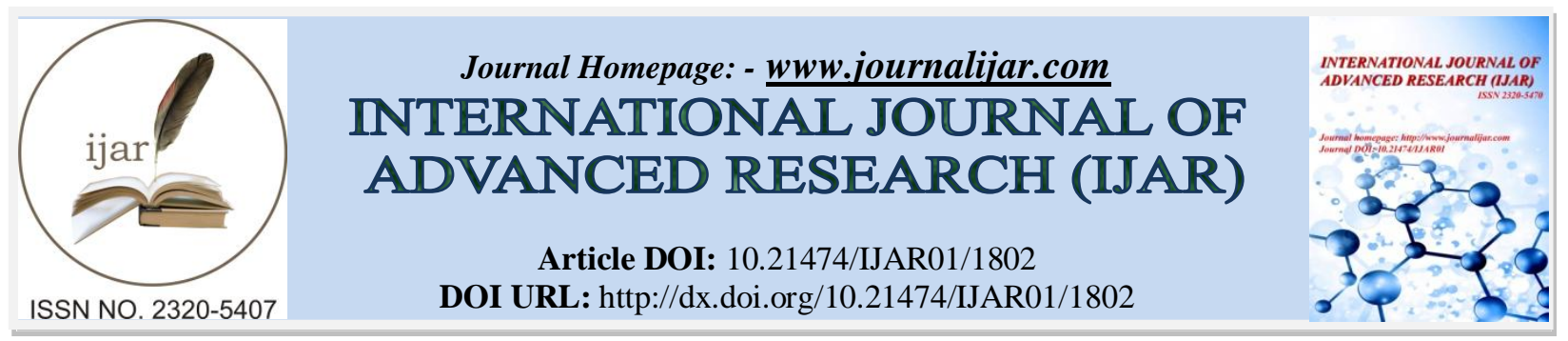

RESEARCH ARTICLE

\title{
OPINIONS OF STUDENTS OF AGRICULTURAL COLLEGE, BAPATLA, TOWARDS TEACHING- LEARNING STYLES.
}

\author{
U. Kiran, T. Prasantha Kumar and T. Gopi Krishna
}

Department of Agricultural Extension, Agricultural College, Bapatla - 522101 Andhra Pradesh.

\section{Manuscript Info}

Manuscript History

Received: 12 August 2016

Final Accepted: 22 September 2016

Published: October 2016

Key words:-

Opinion, students, teaching learning styles.

\section{Abstract}

Agricultural College, Bapatla was purposively selected out of the six Agricultural College in ANGRAU to know the opinions of students and teachers towards teaching-learning styles. The study was conducted by adopting the Ex - post - facto research design. The respondents for the study all final year B. Sc (Ag.) students (90) on rolls as on the date of study in the selected campus due to they had undergone all the courses in different classrooms i.e. ordinary and model classroom The findings of the study revealed that majority of the respondent Students had neutral opinion towards teaching learning styles.

Copy Right, IJAR, 2016,. All rights reserved.

\section{Introduction:-}

Teaching is as old as mankind. The ultimate goal of teaching is to achieve mastery in learning and improve the quality of human performance. Davies (1981) stated that successful teaching is nine tenths perspiration and onetenth inspiration. In other words, besides the art of delivery, the teacher must put in lot of hard work for achieving success in teaching. Teaching cannot happen without learners. In fact, teaching and learning are the two sides of the same coin. A student and teacher's general performance in learning and teaching is influenced by various internal and external factors. The opinions of students towards the model class room collected in the present study are of instrumental Value in improving the model classroom conditions there by improving the learning out come of the s tudent.

\section{Materials and methods:-}

The present Study was conducted by adopting Ex - Post - Facto Research Design. by selecting Agricultural College; Bapatla purposively out of the six Agricultural College in ANGRAU, Andhra Pradesh as it is the oldest College with supposed to be well experienced faculty and required Infrastructural facilities. All Final year B. Sc (Ag.) students (90) were purposively selected. Frequency (f) and Percentages (\%) were used for statistical analysis.

\section{Results and Discussion:-}

Opinion of students towards learning styles:-

Learning styles:-

Learning styles of respondent students was operationally defined as their learning preferences in general as well as their personality dimensions including extra version Vs introversion, sensing Vs intuition, thinking Vs feeling, judging Vs perception over demarcated categories namely independent, dependent, collaborative, competitive, participative and avoidant. 
Distribution of respondent students according to their learning styles.

Table1: Distribution of respondent students according to their learning styles.

$\left(\mathbf{N}_{2}=90\right)$

\begin{tabular}{|c|c|c|c|c|c|c|c|c|c|c|c|}
\hline \multirow{3}{*}{ S.No. } & \multirow{3}{*}{ Item } & \multicolumn{10}{|c|}{ Response Categories } \\
\hline & & \multicolumn{2}{|c|}{$\begin{array}{l}\text { Strongly } \\
\text { Agree }\end{array}$} & \multicolumn{2}{|c|}{ Agree } & \multicolumn{2}{|c|}{ Undecided } & \multicolumn{2}{|c|}{ Disagree } & \multicolumn{2}{|c|}{$\begin{array}{l}\text { Strongly } \\
\text { Disagree }\end{array}$} \\
\hline & & $\mathbf{F}$ & $\%$ & $\mathbf{F}$ & $\%$ & $\mathbf{F}$ & $\%$ & $\mathbf{F}$ & $\%$ & $\mathbf{F}$ & $\%$ \\
\hline 1. & I involve my self while learning. & 35 & 38.88 & 21 & 23.33 & 19 & 21.11 & 13 & 14.44 & 2 & 2.22 \\
\hline 2. & $\begin{array}{l}\text { I learn things open minded enough } \\
\text { without any bias. }\end{array}$ & 28 & 31.11 & 18 & 20 & 30 & 33.33 & 13 & 14.44 & 1 & 1.11 \\
\hline 3. & I am critical, analytical in my learning. & 22 & 24.44 & 9 & 10 & 24 & 26.66 & 35 & 38.88 & --- & --- \\
\hline 4. & I learn by doing. & 29 & 32.22 & 17 & 18.88 & 14 & 15.55 & 30 & 33.33 & 4 & 4.44 \\
\hline 5. & I prefer experiential learning. & 11 & 12.22 & 17 & 18.88 & 17 & 18.88 & 44 & 48.88 & --- & --- \\
\hline 6. & I prefer impartial learning. & 32 & 35.55 & 15 & 16.66 & 6 & 6.66 & 37 & 41.11 & --- & --- \\
\hline 7. & I take risks while learning a thing. & 31 & 34.44 & 25 & 27.77 & 19 & 21.11 & 15 & 16.66 & --- & --- \\
\hline 8. & I am theoretically biased in my learning. & 25 & 27.77 & 18 & 20 & 23 & 25.55 & 24 & 26.66 & --- & --- \\
\hline 9. & I am practically biased in my learning. & 26 & 28.88 & 13 & 14.44 & 7 & 7.77 & 44 & 48.88 & $\begin{array}{c}-- \\
\end{array}$ & --- \\
\hline \multirow{3}{*}{ S.No. } & \multirow{3}{*}{ Item } & \multicolumn{10}{|c|}{ Response Categories } \\
\hline & & \multicolumn{2}{|c|}{$\begin{array}{l}\text { Strongly } \\
\text { Agree }\end{array}$} & \multicolumn{2}{|c|}{ Agree } & \multicolumn{2}{|c|}{ Undecided } & \multicolumn{2}{|c|}{ Disagree } & \multicolumn{2}{|c|}{$\begin{array}{l}\text { Strongly } \\
\text { Disagree }\end{array}$} \\
\hline & & $\mathbf{F}$ & $\%$ & $\mathbf{F}$ & $\%$ & $\mathbf{F}$ & $\%$ & $\mathbf{F}$ & $\%$ & $\mathbf{F}$ & $\%$ \\
\hline 10. & I prefer future oriented learning. & 35 & 38.88 & 13 & 14.44 & 7 & 7.77 & 35 & 38.88 & --- & --- \\
\hline 11. & $\begin{array}{l}\text { I go with a contention that people learn } \\
\text { differently. }\end{array}$ & 41 & 45.55 & 26 & 28.88 & 14 & 15.55 & 9 & 10 & --- & --- \\
\hline 12. & I learn from my colleagues & 42 & 46.66 & 17 & 18.88 & 11 & 12.22 & 19 & 21.11 & 1 & 1.11 \\
\hline 13. & I learn by exposure to mass media. & 25 & 27.77 & 20 & 22.22 & 22 & 24.44 & 24 & 26.66 & 1 & 1.11 \\
\hline 14. & I learn by trail and error method. & 31 & 34.44 & 21 & 23.33 & 25 & 27.77 & 10 & 11.11 & 4 & 4.44 \\
\hline
\end{tabular}

$\mathrm{F}=$ Frequency,$\%=$ Percentage

$\rightarrow$ Multiple response possible

The Table 1 revealed that the percentage of respondent students who expressed their feeling of strongly agree towards different learning styles namely : I involve my self while learning (38.88\%), I learn things open minded enough without any bias (31.11\%), I am critical, analytical in my learning (24.44\%), I learn by doing (32.22\%), I prefer experiential learning (12.22\%), I prefer impartial learning (35.55\%), I take risks while learning a thing (34.44\%), I am theoretically biased in my learning (27.77\%), I am practically biased in my learning (28.88\%), I prefer future oriented learning (38.88\%), I go with a contention that people learn differently $(45.55 \%)$, I learn from my colleagues $(46.66 \%)$, I learn by exposure to mass media $(27.77 \%)$, I learn by trail and error method $(34.44 \%)$.

The percentage of respondent students who expressed their feeling of agree towards different learning styles namely : I involve my self while learning (23.33\%), I learn things open minded enough without any bias (20\%), I am critical, analytical in my learning (10\%), I learn by doing (18.88\%), I prefer experiential learning (18.88\%), I prefer impartial learning (16.66\%), I take risks while learning a thing $(27.77 \%)$, I am theoretically biased in my learning (20\%), I am practically biased in my learning (14.44\%), I prefer future oriented learning (14.44\%), I go with a contention that people learn differently $(28.88 \%)$, I learn from my colleagues (18.88\%), I learn by exposure to mass media $(22.22 \%)$ I learn by trail and error method $(23.33 \%)$.

The percentage of respondent students who unable to decide their feeling towards different learning styles namely : I involve my self while learning (21.11\%), I learn things open minded enough without any bias (33.33\%), I am critical, analytical in my learning (26.66.\%), I learn by doing (15.55\%), I prefer experiential learning (18.88\%), I prefer impartial learning $(6.66 \%)$, I take risks while learning a thing $(21.11 \%)$, I am theoretically biased in my learning (25.55\%), I am practically biased in my learning (7.77\%), I prefer future oriented learning (7.77\%), I go with a contention that people learn differently $(15.55 \%)$, I learn from my colleagues (12.22\%), I learn by exposure to mass media (24.44\%), I learn by trail and error method $(27.77 \%)$. 
The percentage of respondent students who expressed their feeling of disagree towards different learning styles namely : I involve my self while learning (14.44\%), I learn things open minded enough without any bias (14.44\%), I am critical, analytical in my learning (38.88\%), I learn by doing (33.33\%), I prefer experiential learning (48.88\%), I prefer impartial learning $(41.11 \%)$, I take risks while learning a thing $(16.66 \%)$, I am theoretically biased in my learning (26.66\%), I am practically biased in my learning (48.88\%), I prefer future oriented learning (38.88\%), I go with a contention that people learn differently (10\%), I learn from my colleagues $(21.11 \%)$, I learn by exposure to mass media (26.66\%), I learn by trail and error method $(11.11 \%)$.

The percentage of respondent students who expressed their feeling of strongly disagree towards different learning styles namely : I involve my self while learning $(2.22 \%)$, I learn things open minded enough without any bias $(1.11 \%)$, I learn by doing (4.44\%), I learn from my colleagues $(1.11 \%)$, I learn by exposure to mass media (1.11\%), I learn by trail and error method $(4.44 \%)$.

In nutshell majority of the students preferred the learning styles viz., I involve my self while learning, I learn things open minded enough without any bias, I learn by doing, I prefer impartial learning, I take risks while learning a thing, I prefer future oriented learning, I go with a contention that people learn differently, I learn from my colleagues, I learn by exposure to mass media, I learn by trail and error method. The above findings were are inline with the findings of Vijayabhinandan (2003).

\section{Perception of students towards teaching styles:-}

Students perception towards teaching style define as psychological perception i.e. personal feeling towards different teaching styles.

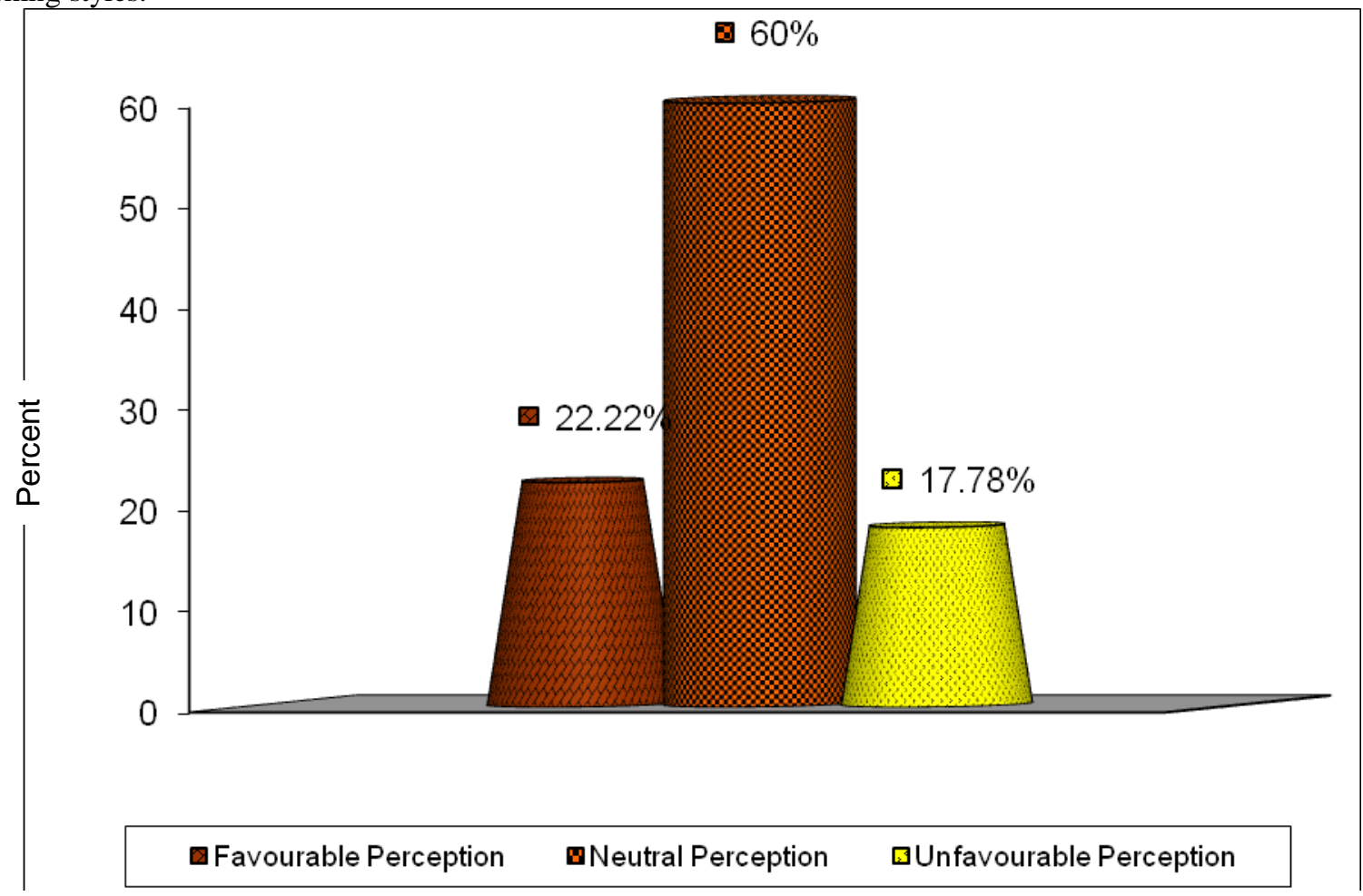

Fig 1:- Distribution of respondent students according to their perception towards teaching styles.

Table 2:- Distribution of respondent students according to their perception towards teaching styles.

\begin{tabular}{|c|l|c|c|}
\hline \multicolumn{1}{|c|}{ Category } & \multicolumn{2}{c|}{$\left.\mathbf{N}_{\mathbf{2}}=\mathbf{9 0}\right)$} \\
\cline { 3 - 4 } S.No. & & Frequency & Percentage \\
\hline 1. & Favourable Perception & 20 & 22.22 \\
\hline 2. & Neutral Perception & 54 & 60.00 \\
\hline 3. & Unfavourable Perception & 16 & 17.78 \\
\hline
\end{tabular}


From Table 2 inferred that vast majority (60\%) of the students had neutral perception towards teaching students, 22.22 per cent had favourable perception and 17.78 per cent had unfavourable perception towards teaching styles. This was shown in Fig 1. It was inline with the findings of Reddy (2002).

\section{Bibliography:-}

1. Davies Kelvin Ivor 1981 Instructional Technique Mc Graw Hill Book Company, New York.

2. Options in Classroom Technology for the Year 2000, Larry Gilbert, January, 1999. E-mail: Larry.Gilbert@wwu.edu.

3. Owu C 1992 Learning Styles of Post secondary Students Enrolled in Vocational Technical Institutes. Journal of Industrial Teacher Education 36 (4): National Association of Industrial and Technical Teacher Educators (ISSN 0022-1864).

4. www.classrooms.com.

5. Vijayabhinandan 2003 Teaching and learning styles of teachers and students of ANGRAU, Ph.D. Thesis submitted to ANGRAU Hyderabad

6. Reddy $\mathbf{K} \mathbf{G} \mathbf{2 0 0 2}$ Training environment as perceived by the trainers of agricultural training institutes unpublished Ph.D. Thesis submitted to ANGRAU, Hyderabad. 